\title{
Analysis of Packet Combining for Single Carrier Multi-Relay Broadband System
}

\author{
Houda Chafnaji ${ }^{\ddagger *}$, Tarik Ait-Idir ${ }^{\ddagger *}$, Halim Yanikomeroglu ${ }^{+}$, and Samir Saoudi* \\ ${ }^{\ddagger}$ Communications Systems Department, INPT, Madinat Al Irfane, Rabat, Morocco \\ ${ }^{*}$ INSTITUT TELECOM/ TELECOM Bretagne, Signal and Communications Department, CS 83818, 29238 Brest Cedex, France. \\ + Broadband Communications and Wireless Systems (BCWS) Centre, \\ Department of Systems and Computer Engineering, Carleton University, Ottawa, Canada \\ Emails: houda.chafnaji,samir.saoudi@telecom-bretagne.eu, aitidir@ieee.org, halim@sce.carleton.ca
}

\begin{abstract}
This paper focuses on packet combining for multi-relay systems operating over multiple-input-multiple-output (MIMO) broadband channel. The work presented in this paper is valid for both amplify-andforward (AF) and decode-and-forward (DF) relaying schemes. First, we drive a communication model in such a way that the destination can see the received signals during the relaying slots as direct retransmissions from the source. We then present a multi-slot communication model where transmission over relaying slots is translated into virtual receive antennas and examine the outage probability of different relaying schemes. Using simulations, we show that this schemes outperform each other depending on the relays location and demonstrate that the multirelay transmissions provide better diversity gain than the conventional hybrid-Automatic repeat request (ARQ).
\end{abstract}

Index Terms-Cooperative relaying, multiple-antenna systems, packet combining, outage probability.

\section{INTRODUCTION}

In wireless networks, the presence of diversity between the source and the destination is a key requirement to combat channel fading and enable communication at high spectral efficiencies. In this paper, we concentrate on temporal and spatial diversity. ARQ combined with forward error correction (FEC) is a popular mechanism to exploit temporal diversity. This mechanism has been studied for many years and is still receiving considerable attention [1], [2]. However, it suffers from temporal diversity limitations especially in slow fading environments. In [3], the authors have proposed cooperative relaying transmission as a solution for mitigating these diversity limitations. This cooperative diversity version exploits the broadcast nature of the wireless channel and adds spatial diversity by incorporating relays in the network. The relays play the role of packet retransmitters instead of the source, thereby creating an independent channel to increase the diversity order. Cooperative relaying presents a good alternative to classical ARQ and is becoming an area of wide interest for many researchers (see for instance [4], [5]). In this new transmission mechanism, one or more relays assist the communication between the source and destination to form a MIMO system and therefore build up space-time diversity branches that are exploited at the destination.

Several interesting relaying schemes have been proposed, among which are two basic modes: amplify-and-forward (AF), and decodeand-forward (DF). The AF strategy represents the simplest way that a relay may cooperate with the source and the destination. Under this scheme, the relay simply amplifies the received signal and forwards it towards the destination. However, in the DF scheme, the relay first decodes the signal received from the source, re-encodes and retransmits it to the destination. This approach suffers from error propagation when the relay transmits an erroneously decoded data block [6], [7]. Selective DF, where the relay only transmits when it can reliably decode the data packet, has been introduced as an efficient method to reduce error propagation [8]. However, the unsuccessful detection of the data packet by one or more relays can limit the benefit of relaying. In fact, for each incorrectly detected packet, there is a waste of one time slot. To prevent the incurring "silence", a modified selective DF scheme has been proposed in [6], [9]. In this scheme, when the relay fails to correctly decode the packet, it sends back a negative acknowledgment (NACK) message to the source that directly transmits the packet to the destination during the slot allocated to the relay. In this paper, we refer to this scheme as ACK/NACK-aided DF.

In this paper, we consider a broadband multi-relay system operating under the framework of protocol II where the source broadcasts the data packet to both the relay and the destination during the first slot while during the second slot only the relay sends the packet to the destination[10]. We focus on cooperative ARQ communication where the feedback from the destination is exploited and the packet repetition is activated only if the destination fails to decode the data packet [11], [12]. First, we derive an unified communication model for the relaying schemes under consideration in this paper, i.e., AF, Selective DF, and ACK/NACK-aided DF. We then derive a multi-slots communication model that helps, at the destination side, to perform the combination of packets received over multiple slots. We also investigate the outage probability of different relaying schemes. To the best of the authors knowledge, previous works that addressed outage analysis of relay communication systems have focused on the case of single relay transmissions (see for instance [8] and [13]). In this paper, we provide outage analysis of multirelay multi-slot cooperative ARQ. Using simulations, we show that the studied relaying schemes outperform each other depending on relay locations and demonstrate that the multi-relay transmissions provide better diversity gain than the conventional hybrid-ARQ. Throughout the paper we use the following notation: $(.)^{\top}$ and $(.)^{\mathrm{H}}$ are the transpose and the transpose conjugate of the argument, respectively. $\emptyset$ denotes the empty set. $\operatorname{diag}\left\{\mathbf{X}_{1}, \cdots, \mathbf{X}_{m}\right\}$ denote the block diagonal matrix constructed from $\mathbf{X}_{1}, \cdots, \mathbf{X}_{m} \in \mathbb{C}^{n_{1} \times n_{2}}$, respectively. For $\mathbf{x} \in \mathbb{C}^{T N}, \mathbf{x}_{f}$ denotes the discrete Fourier transform (DFT) of $\mathbf{x}$, i.e. $\mathbf{x}_{f}=\mathbf{U}_{T, N} \mathbf{x}$, with $\mathbf{U}_{T, N}=\mathbf{U}_{T} \otimes \mathbf{I}_{N}$, where $\mathbf{I}_{N}$ is the $N \times N$ identity matrix, $\mathbf{U}_{T}$ is a unitary $T \times T$ matrix whose $(m, n)$ th element is $\left(\mathbf{U}_{T}\right)_{m, n}=\frac{1}{\sqrt{T}} e^{-j(2 \pi m n / T)}, j=\sqrt{-1}$, and $\otimes$ denotes the Kronecker product.

The remainder of the paper is organized as follows: In Section II, we introduce the unified communication model for the relaying schemes together with the multi-slot block communication model. In Section III, we investigate the outage probability of the considered relaying schemes. Outage analysis is provided in Section IV. Finally, the paper is concluded in Section V. 


\section{RELAY SYSTEM MODEL}

\section{A. Multi-Relay Transmission Scheme}

We consider a multi-relay-assisted wireless communication system, where the $M_{\mathrm{S}}$ antenna source terminal denoted as $\mathrm{S}$ transmits information blocks to the $M_{\mathrm{D}}$ antenna destination terminal denoted as $\mathrm{D}$ with the assistance of $K-1$ dedicated relays denoted as $\mathrm{R}_{2}, \cdots, \mathrm{R}_{k}, \cdots, \mathrm{R}_{K}$. Each relay $\mathrm{R}_{k}$ is equipped with $M_{\mathrm{R}_{k}}$ transmit and receive antennas. We consider a relaying system using up to $K$ slots for sending one information block from the source to the destination, where each slot spans $T$ channel uses. During the first slot, the source broadcasts the data packet to the $K-1$ relays and the destination. During the following slots, each relay participates to the packet retransmission during the allocated slot and keeps silent during the other slots. In this work, we focus on cooperative ARQ communication where the feedback from the destination is exploited and packet retransmission is activated only if the destination fails to decode the data packet. Therefore, during the relaying slots, once decoding is successful, the destination broadcasts a positive acknowledgment (ACK) to the source and the relays to stop relaying the current block and move on to the next information block. In this work, we suppose perfect packet error detection and assume that the one bit ACK/NACK feedback message is errorfree. The source-relay ( $\rightarrow \mathrm{R}_{k}$ ), source-destination $(\mathrm{S} \rightarrow \mathrm{D})$, and relay-destination $\left(\mathrm{R}_{k} \rightarrow \mathrm{D}\right)$ links are assumed to be frequency selective. The channel matrices corresponding to the $A \rightarrow B$ link are $\mathbf{H}_{0}^{(A B)}, \cdots, \mathbf{H}_{L_{A B}-1}^{(A B)} \in \mathbb{C}^{M_{B} \times M_{A}}$ with $L_{A B}$ denotes the number of symbol-spaced taps, $A \in\left\{\mathrm{S}, \mathrm{R}_{k}\right\}$, and $B \in\left\{\mathrm{R}_{k}, \mathrm{D}\right\}$. Their entries are zero-mean circularly symmetric complex Gaussian random variables. Cyclic prefix (CP)-aided transmission is assumed for all links. This prevents inter-block interference and allows the use of frequency domain processing at the receiver side. The average energies of the different links are $E_{\mathrm{SR}_{k}}, E_{\mathrm{SD}}$ and $E_{\mathrm{R}_{k} \mathrm{D}}$, and take into account the path-loss and shadowing effects of each link. We suppose that no channel state information at the transmitter (CSIT) is available and assume perfect channel state information (CSI) at the relays and the destination.

First, the source encodes its data blocks using a space-time bit interleaved coded modulation (STBICM) encoder. The resulting symbol vector, at the output of the STBICM encoder, is given by,

$$
\mathbf{s} \triangleq\left[\mathbf{s}_{0}^{\top}, \cdots, \mathbf{s}_{T-1}^{\top}\right]^{\top} \in \mathcal{S}^{M_{S} T}
$$

where $\mathbf{s}_{i} \triangleq\left[s_{1, i}^{\top}, \cdots, s_{t, i}^{\top}, \cdots, s_{M_{\mathrm{S}, i}}^{\top}\right]^{\top} \in \mathcal{S}^{M_{\mathrm{S}}}$ is the symbol vector at channel use $i=0, \cdots, T-1$, and $\mathcal{S}$ is the symbol constellation set. During the first slot, the source inserts a CP symbol word of length $T_{C P}^{\mathrm{S} \rightarrow \mathrm{D}} \geq \max _{k=2, \cdots, K}\left(L_{\mathrm{SR}_{k}}, L_{\mathrm{SD}}\right)$, then broadcasts the resulting symbol frame. After $\mathrm{CP}$ deletion, the baseband $M_{\mathrm{D}} \times 1$ signal vector obtained at the destination side is given by,

$$
\mathbf{y}_{i}^{(1)}=\sqrt{E_{\mathrm{SD}}} \sum_{l=0}^{L_{\mathrm{SD}}-1} \mathbf{H}_{l}^{(1)} \mathbf{s}_{(i-l) \bmod T}+\mathbf{n}_{i}^{(1)},
$$

where $\mathbf{H}_{l}^{(1)}=\mathbf{H}_{l}^{(\mathrm{SD})}$, and $\mathbf{n}_{i}^{(1)} \sim \mathcal{N}\left(\mathbf{0}_{M_{\mathrm{D}} \times 1}, \sigma^{2} \mathbf{I}_{M_{\mathrm{D}}}\right)$ is the thermal noise. During the following $K-1$ slots, the transmission strategy depends on the considered relaying scheme. After CP deletion, The $M_{\mathrm{D}} \times 1$ received signal vector at the destination side, at each slot $k=2, \ldots, K$ can be expressed as

$$
\mathbf{y}_{i}^{(k)}=\sqrt{E_{k}} \sum_{l=0}^{L_{k}-1} \mathbf{H}_{l}^{(k)} \mathbf{s}_{(i-l) \bmod T}+\mathbf{n}_{i}^{(k)} .
$$

This expression is valid for both AF and DF schemes:
- In AF scheme, each relay $\mathrm{R}_{k}$ amplifies and sends the block of received signals to the destination during the allocated slot. In this case, $\mathbf{H}_{l}^{(k)}$ in (3) is the $l$ th equivalent tap channel matrix corresponding to link $\mathrm{S} \rightarrow \mathrm{R}_{k} \rightarrow \mathrm{D}$. It is the convolution of the two channels corresponding to links $\mathrm{S} \rightarrow \mathrm{R}_{k}$ and $\mathrm{R}_{k} \rightarrow \mathrm{D}$. $L_{k}=L_{\mathrm{SR}_{k}}+L_{\mathrm{R}_{k} \mathrm{D}}-1$ is the equivalent channel order, and $E_{k}=\frac{E_{\mathrm{R}_{k} \mathrm{D}} E_{\mathrm{SR}_{k}}}{M_{\mathrm{S}} E_{\mathrm{SR}_{k}}+\sigma^{2}}[14]$.

- In DF schemes, the relays transmit using $M_{\mathrm{S}}$ antennas. In the case of selective DF, $\mathbf{H}_{l}^{(k)}=\mathbf{H}_{l}^{\left(\mathrm{R}_{k} \mathrm{D}\right)} \in \mathbb{C}^{M_{\mathrm{D}} \times M_{\mathrm{S}}}$, $L_{k}=L_{\mathrm{R}_{k} \mathrm{D}}$, and $E_{k}=E_{\mathrm{R}_{k} \mathrm{D}}$ if relay $k$ can correctly decode the packet, otherwise, the packet retransmission is not activated during slot $k$. When ACK/NACK-aided DF is considered, both the relay and the source are involved during the following $K-1$ slots. In fact, at each relay, an acknowledgment message is generated after the information block decoding. If the decoding outcome is erroneous, the relay broadcasts a NACK message to both the destination and the source to indicate that during the allocated slot the source is going to directly send the symbol frame to the destination. In this case, $\mathbf{H}_{l}^{(k)}=\mathbf{H}_{l}^{(\mathrm{SD})}, L_{k}=L_{\mathrm{SD}}$, and $E_{k}=E_{\mathrm{SD}}$. The reception of an ACK message indicates that during the allocated slot, the source will keep silent while the relay will transmit the symbol frame to the destination, which means $\mathbf{H}_{l}^{(k)}=\mathbf{H}_{l}^{\left(\mathrm{R}_{k} \mathrm{D}\right)} \in \mathbb{C}^{M_{\mathrm{D}} \times M_{\mathrm{S}}}, L_{k}=L_{\mathrm{R}_{k} \mathrm{D}}$, and $E_{k}=E_{\mathrm{R}_{k} \mathrm{D}}$.

\section{B. Multi-Slot Block Communication Model}

Using the signal communication model (3), derived in the previous section, the received signals during the relaying slots can be viewed as a direct retransmissions from the source as it is shown in Fig. 1. In fact, (3) is of a great importance as it allows us to view each relaying slot as an additional set of virtual receive antennas at the destination side. Therefore, after $k$ slots, the system (source, $k-1$ relays, and destination) can be viewed as a point to point MIMO link with $M_{\mathrm{S}}$ transmit and $k M_{\mathrm{D}}$ receive antennas. First, we introduce

$$
\underline{\mathbf{y}}_{i}^{(k)} \triangleq\left[\mathbf{y}_{i}^{(1)^{\top}}, \cdots, \mathbf{y}_{i}^{(k)^{\top}}\right]^{\top} \in \mathbb{C}^{k M_{\mathrm{D}}}
$$

where reception over multiple slots can be viewed as multi-antenna reception. We construct the $k M_{\mathrm{D}} T \times 1$ block received signal vector $\underline{\mathbf{y}}^{(k)}$ as,

$$
\underline{\mathbf{y}}^{(k)} \triangleq\left[\underline{\mathbf{y}}_{0}^{(k)^{\top}}, \cdots, \underline{\mathbf{y}}_{T-1}^{(k)^{\top}}\right]^{\top} \in \mathbb{C}^{k M_{\mathrm{D}} T} .
$$

The block communication model corresponding to this $k$-slot scheme is given by,

$$
\underline{\mathbf{y}}^{(k)}=\underline{\mathcal{H}}^{(k)} \mathbf{s}+\underline{\mathbf{n}}^{(k)},
$$

where $\underline{\mathcal{H}}^{(k)} \in \mathbb{C}^{k M_{\mathrm{D}} T \times M_{\mathrm{S}} T}$ is a block circulant matrix whose first $k M_{\mathrm{D}} T \times M_{\mathrm{S}}$ block column matrix is

$$
\left[\underline{\mathbf{H}}_{0}^{(\mathrm{k})^{\top}}, \cdots, \underline{\mathbf{H}}_{L-1}^{(\mathrm{k})^{\top}}, \mathbf{0}_{(T-L) k M_{\mathrm{D}} \times M_{\mathrm{S}}}\right]^{\top},
$$

with

$$
\left\{\begin{array}{l}
L=\max _{k=1, \cdots, K}\left(L_{k}\right), \\
\underline{\mathbf{H}}_{l}^{(k)} \triangleq\left[\sqrt{E_{1}} \mathbf{H}_{l}^{(1)^{\top}}, \cdots, \sqrt{E_{k}} \mathbf{H}_{l}^{(k)^{\top}}\right]^{\top} \in \mathbb{C}^{k M_{\mathrm{D}} \times M_{\mathrm{S}}}
\end{array}\right.
$$

correspond to the order and the lth tap of the virtual MIMO channel, respectively. Vector

$$
\underline{\mathbf{n}}^{(k)}=\left[\underline{\mathbf{n}}_{0}^{(k)^{\top}}, \cdots, \underline{\mathbf{n}}_{T-1}^{(k)^{\top}}\right]^{\top} \in \mathbb{C}^{k M_{\mathrm{D}} T},
$$




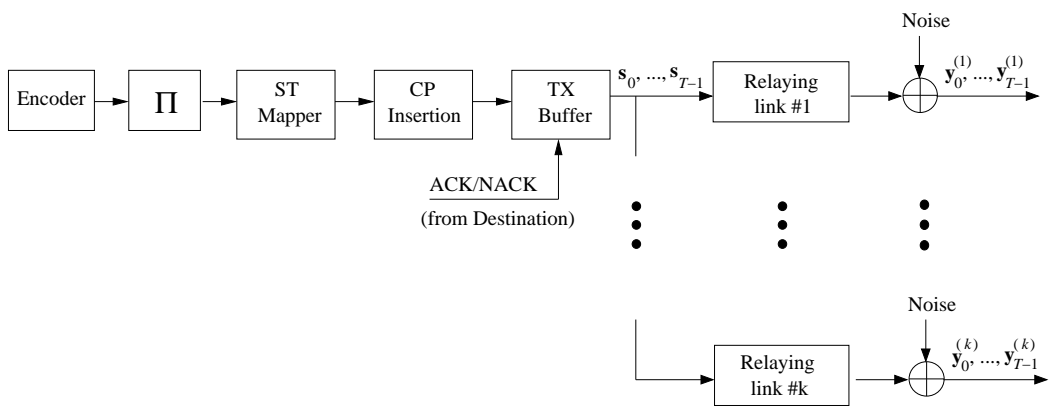

Figure 1. ST-BICM diagram for multi-relay assisted systems.

denotes the thermal noise present in the $k$-slot equivalent MIMO system, where $\underline{\mathbf{n}}_{i}^{(k)} \triangleq\left[\mathbf{n}_{i}^{(1)^{\top}}, \cdots, \mathbf{n}_{i}^{(k)^{\top}}\right]^{\top} \sim$ $\mathcal{N}\left(\mathbf{0}_{k M_{\mathrm{D}} \times 1}, \sigma^{2} \mathbf{I}_{k M_{\mathrm{D}}}\right)$. Note that the block circulant matrix $\underline{\mathcal{H}}^{(k)}$ can be block diagonalized in a Fourier basis as

$$
\underline{\mathcal{H}}^{(k)}=\mathbf{U}_{T, k M_{\mathrm{D}}}^{\mathrm{H}} \underline{\boldsymbol{\Lambda}}^{(k)} \mathbf{U}_{T, M_{\mathrm{S}}} .
$$

Therefore, applying the DFT to the $k$-slot block signal vector (6) yields the following frequency domain block communication model,

$$
\underline{\mathbf{y}}_{f}^{(k)}=\underline{\Lambda}^{(k)} \mathbf{s}_{f}+\underline{\mathbf{n}}_{f}^{(k)},
$$

where

$$
\left\{\begin{array}{l}
\underline{\boldsymbol{\Lambda}}^{(k)} \triangleq \operatorname{diag}\left\{\underline{\boldsymbol{\Lambda}}_{0}^{(k)}, \cdots, \underline{\boldsymbol{\Lambda}}_{T-1}^{(k)}\right\} \in \mathbb{C}^{k M_{\mathrm{D}} \times M_{\mathrm{S}} T}, \\
\underline{\boldsymbol{\Lambda}}_{i}^{(k)}=\sum_{l=0}^{L-1} \underline{\mathbf{H}}_{l}^{(k)} e^{-j(2 \pi i l / T)} \in \mathbb{C}^{k M_{\mathrm{D}} \times M_{\mathrm{S}}},
\end{array}\right.
$$

and $\mathbf{s}_{f}$ and $\underline{\mathbf{n}}_{f}^{(k)}$ are the DFT of $\mathbf{s}$ and $\underline{\mathbf{n}}^{(k)}$, respectively.

\section{OUtage Probability}

The outage probability is regarded as a meaningful tool for evaluating the performance of non-ergodic channels, i.e., block fading quasi-static channels, as it provides a lower bound on the block error rate (BLER) [15, p. 187]. For a given signal to noise ratio (SNR) $\gamma$ per receive antenna, the outage probability of the direct link (i.e., $\mathrm{S} \rightarrow \mathrm{D}$ link), at a target transmission rate $\mathcal{R}$, is defined as the probability that the mutual information $I$ between the transmitter alphabet and the received signal is below $\mathcal{R}$,

$$
P_{\text {out }}^{\text {Direct }}(\mathcal{R}, \gamma, k=1)=\operatorname{Pr}\left\{I\left(\mathbf{s}_{f}, \mathbf{y}_{f}^{(1)} \mid \boldsymbol{\Lambda}^{(1)}, \gamma\right)<\mathcal{R}\right\},
$$

where $\mathbf{s}_{f}$ is the frequency domain transmitted symbol vector, $\mathbf{y}_{f}^{(1)}$ is the frequency domain received signal over link $\mathrm{S} \rightarrow \mathrm{D}$ (during slot $k=1$ ), and $\boldsymbol{\Lambda}^{(1)}$ is the corresponding channel frequency response (CFR). In this work, we are interested in analyzing cooperative ARQ communications where packet relaying is activated only if the destination fails to decode the initially transmitted data packet. In such a scenario, packet combining starts when the direct link is in outage, i.e., $k \geq 2$.

At each slot $k \geq 2, k$ copies of the transmitted packet are available at the destination side, one from the direct link and $k-1$ from relaying links, except for selective DF scheme where the number of transmitted packet copies depends on the quality of $S \rightarrow R$ link realizations. In fact, for selective $\mathrm{DF}$, packet retransmission does not occur at slot $u(2 \leq u \leq k)$ if the $\mathrm{S} \rightarrow \mathrm{R}_{u}$ link is in outage. However, for the sake of simplicity, we assume that slot $u$ is allocated for packet retransmission even when relaying is deactivated. Therefore, the $k$-slot relaying system can be viewed as a repetition coding scheme where $k$ parallel sub-channels are used to transmit one symbol message [15, p. 194]. Using the unified communication model (11), the outage probability of the studied relaying schemes can be expressed as in [16], i.e.,

$$
\begin{aligned}
& P_{\text {out }}(\mathcal{R}, \gamma, k)= \\
& \quad \operatorname{Pr}\left\{\frac{1}{k} I\left(\mathbf{s}_{f}, \underline{\mathbf{y}}_{f}^{(k)} \mid \underline{\boldsymbol{\Lambda}}^{(k)}, \gamma\right)<\mathcal{R}, \mathcal{A}_{1}, \cdots, \mathcal{A}_{k-1}\right\},
\end{aligned}
$$

where $\mathcal{A}_{u}$ denotes the event that the destination sends a NACK message at slot $u$. In the case of independent and identically distributed (i.i.d) circularly symmetric complex channel inputs, the mutual information $I\left(\mathbf{s}_{f}, \underline{\mathbf{y}}_{f}^{(k)} \mid \underline{\boldsymbol{\Lambda}}^{(k)}, \gamma\right)$ in (14) can be expressed as in [17], i.e.,

$$
\begin{aligned}
I\left(\mathbf{s}_{f}, \underline{\mathbf{y}}_{f}^{(k)} \mid\right. & \left.\underline{\boldsymbol{\Lambda}}^{(k)}, \gamma\right)= \\
& \frac{1}{T} \sum_{i=0}^{T-1} \log _{2}\left(\operatorname{det}\left(\mathbf{I}_{k M_{\mathrm{D}}}+\frac{\gamma}{M_{\mathrm{S}}} \underline{\boldsymbol{\Lambda}}_{i}^{(k)} \underline{\boldsymbol{\Lambda}}_{i}^{(k)^{\mathrm{H}}}\right)\right) .
\end{aligned}
$$

Moreover, in the special case of DF schemes, the outage probability can be expressed using the outage probabilities of $\mathrm{S} \rightarrow \mathrm{R}$ links. For DF modes, the received signals available at the destination side after $k$ slots, depend on the quality of $\mathrm{S} \rightarrow \mathrm{R}$ links. Let $\mathcal{E}_{k}$ denote the event that the relaying system \{Source, $k-1$ relays, destination \} is in outage at slot $k$, and $\mathcal{C}_{k, \tau}$ denote the event that $\tau$ source-relay links among the $k-1$ available source-relay links (i.e., $\mathrm{S} \rightarrow \mathrm{R}_{2}, \cdots, \mathrm{S} \rightarrow$ $\mathrm{R}_{k}$ ) are in outage. The outage probability of the DF relaying system at slot $k$ can be expressed as,

$$
P_{\text {out }}^{\mathrm{DF}}(\mathcal{R}, \gamma, k)=\sum_{\tau=0}^{k-1} \operatorname{Pr}\left\{\mathcal{E}_{k}, \mathcal{C}_{k, \tau}, \mathcal{A}_{1}, \cdots, \mathcal{A}_{k-1}\right\} .
$$

In the case of selective DF mode, packet retransmission does not occur at slot $u(2 \leq u \leq k)$ if the $\mathrm{S} \rightarrow \mathrm{R}_{u}$ link is in outage. Let $\mathbf{R}_{k, \tau} \subset\left\{\mathrm{R}_{2}, \cdots, \mathrm{R}_{k}\right\}$ denote the set of $\tau$ relays involved in event $\mathcal{C}_{k, \tau}$, and $\overline{\mathbf{R}}_{k, \tau} \triangleq\left\{\mathrm{R}_{2}, \cdots, \mathrm{R}_{k}\right\} \backslash \mathbf{R}_{k, \tau}$. For selective DF relaying, the outage probability (16) can then be expressed as,

$$
\begin{aligned}
& P_{\mathrm{out}}^{\mathrm{SDF}}(\mathcal{R}, \gamma, k)= \\
& \sum_{\tau=0}^{k-1} \sum_{\mathbf{R}_{k, \tau}} P_{\mathrm{out}}^{\mathrm{S}, \overline{\mathbf{R}}_{k, \tau} \rightarrow \mathrm{D}} \prod_{\mathrm{R} \in \mathbf{R}_{k, \tau}} P_{\mathrm{out}}^{\mathrm{S} \rightarrow \mathrm{R}} \prod_{\mathrm{R}^{\prime} \in \overline{\mathbf{R}}_{k, \tau}}\left(1-P_{\mathrm{out}}^{\mathrm{S} \rightarrow \mathrm{R}^{\prime}}\right),
\end{aligned}
$$

where $P_{\mathrm{out}}^{\mathrm{S} \rightarrow \mathrm{R}}$ denotes the outage probability of the $\mathrm{S} \rightarrow \mathrm{R}$ link and is computed as,

$$
P_{\text {out }}^{\mathrm{S} \rightarrow \mathrm{R}}=\operatorname{Pr}\left\{\frac{1}{k} I\left(\mathbf{s}_{f}, \mathbf{y}_{f}^{(\mathrm{SR})} \mid \mathbf{\Lambda}^{(\mathrm{SR})}, \gamma\right)<\mathcal{R}, \mathcal{A}_{1}, \cdots, \mathcal{A}_{k-1}\right\} .
$$

In (18), $\mathbf{y}_{f}^{(\mathrm{SR})}$ denotes the frequency domain received signal over the $\mathrm{S} \rightarrow \mathrm{R}$ link, and $\boldsymbol{\Lambda}^{(\mathrm{SR})}$ is the corresponding CFR. While $P_{\text {out }}^{\mathrm{S}, \overline{\mathbf{R}}_{k, \tau} \rightarrow \mathrm{D}}$ is the outage probability of the system after combining at slot $k$ using relays in set $\overline{\mathbf{R}}_{k, \tau}$. It is computed similarly to (14) using $\mathrm{R} \rightarrow \mathrm{D}$ link channel matrices corresponding to relays in set $\overline{\mathbf{R}}_{k, \tau}$. Note that when 
$\mathbf{R}_{k, 0}=\emptyset$ and $\overline{\mathbf{R}}_{k, k-1}=\emptyset$, the corresponding multiplicative terms in (17) are assumed equal to 1 .

In the case of ACK/NACK-aided DF mode, the packet is directly retransmitted by the source during slot $u$ if the link $\mathrm{S} \rightarrow \mathrm{R}_{u}$ is in outage. Let $\mathbf{S}_{k, \tau}$ denote the set of $\tau$ time slots during which the source is involved in the packet retransmission. The outage probability (16) can be expressed as,

$$
\begin{aligned}
& P_{\text {out }}^{\mathrm{ACK} / \mathrm{NACK}-\mathrm{DF}}(\mathcal{R}, \gamma, k)= \\
& \sum_{\tau=0}^{k-1} \sum_{\mathbf{R}_{k, \tau}} P_{\mathrm{out}}^{\mathrm{S}_{k, \tau}, \overline{\mathbf{R}}_{k, \tau} \rightarrow \mathrm{D}} \prod_{\mathrm{R} \in \mathbf{R}_{k, \tau}} P_{\mathrm{out}}^{\mathrm{S} \rightarrow \mathrm{R}} \prod_{\mathrm{R}^{\prime} \in \overline{\mathbf{R}}_{k, \tau}}\left(1-P_{\mathrm{out}}^{\mathrm{S} \rightarrow \mathrm{R}^{\prime}}\right),
\end{aligned}
$$

where $P_{\text {out }}^{\mathbf{S}_{k, \tau}, \overline{\mathbf{R}}_{k, \tau} \rightarrow \mathrm{D}}$ denotes the outage probability of the system after combining at slot $k$ using $\tau$ packet copies from $\mathrm{S} \rightarrow \mathrm{D}$ links and $k-\tau$ copies from $\mathrm{R}^{\prime} \rightarrow \mathrm{D}$ links where $\mathrm{R}^{\prime} \in \overline{\mathbf{R}}_{k, \tau}$.

\section{OUtage Analysis}

In this section, we analyze the outage probability of the studied relaying schemes. We use Monte Carlo simulations to evaluate system outage probability given by (14). First, we generate MIMO channel matrices corresponding to the $\mathrm{S} \rightarrow \mathrm{D}$ link and compute the mutual achievable rate using (15) for $k=1$. If the achievable rate is greater than $\mathcal{R}$, the system is declared in a non outage, relaying is therefore deactivated, and the system moves on to the transmission of the next block. However if the target rate $\mathcal{R}$ is not reached when $k=1$, the first relaying link MIMO channel matrices are generated depending on the relaying scheme in use and the mutual achievable rate is re-calculated for $k=2$. The relaying process is stopped and the processing of the next block transmission is started, either because the achievable rate is greater than $k \mathcal{R}$ at slot $k \leq K$ or the system is in outage, i.e., the achievable rate is below $K \mathcal{R}$ at the last slot $K$. For simulations, we choose $T=258$ channel use. For the sake of simplicity, we assume that all relays are at the same distance from both the source and the destination. We consider a homogeneous case where the distances between the source and relay $l_{\mathrm{SR}}$, relay and destination $l_{\mathrm{RD}}$, and source and destination $l_{\mathrm{SD}}$ are normalized in such a way that $l_{\mathrm{SR}}+l_{\mathrm{RD}}=l_{\mathrm{SD}}=1$. All links have the same frequency-selective fading channel profile, i.e., $L=3$ equal power paths with the same path loss exponent $\kappa=3$. The link average energy is $E_{A B}=\left(l_{A B}\right)^{-\kappa}$ with $A=\mathrm{S}$ or $\mathrm{R}$, and $B=\mathrm{R}$ or $\mathrm{D}$.

First, we consider a one-relay cooperative ARQ system $(K=2$ slots) where all nodes are equipped with two antennas, i.e., $M_{\mathrm{S}}=$ $M_{\mathrm{R}}=M_{\mathrm{D}}=2$ and the target rate is $\mathcal{R}=2$. In Fig. 2, we report the outage probability versus $l_{S R}$ for $\mathrm{S} \rightarrow \mathrm{D}$ link SNR, i.e., $\mathrm{SNR}_{\mathrm{SD}}$ $=3 \mathrm{~dB}$. In the legend, ACK/NACK-DF (Slow Ch) and ACK/NACKDF (fast Ch) denote the ACK/NACK-aided DF scheme operating over an $\mathrm{S} \rightarrow \mathrm{D}$ long-term static channel where the channel is constant over $K$ consecutive slots, and a $\mathrm{S} \rightarrow \mathrm{D}$ short-term static channel where the channel independently changes from slot to slot, respectively. From Fig. 2, we notice that the optimal relay location for all studied schemes is $l_{\mathrm{SR}}=0.5$. Moreover, the results show that $\mathrm{AF}$ and $\mathrm{DF}$ relaying outperform each other depending on the relay location. In fact, AF scheme seems to be more suitable for locations close to the destination, i.e., $l_{\mathrm{SR}}>0.6$. However, for locations close to the source, the relay experiences better radio conditions, and the probability of successful data packet decoding becomes higher. In this case, DF schemes are more suitable. Furthermore, the studied DF schemes have similar performances when $l_{\mathrm{SR}}<0.6$. For relay locations where $l_{\mathrm{SR}}>0.6$, ACK/NACK-aided DF scheme clearly outperforms selective DF scheme. However, when the $\mathrm{S} \rightarrow \mathrm{D}$ link experiences slow fading the gap becomes too small. In this case,

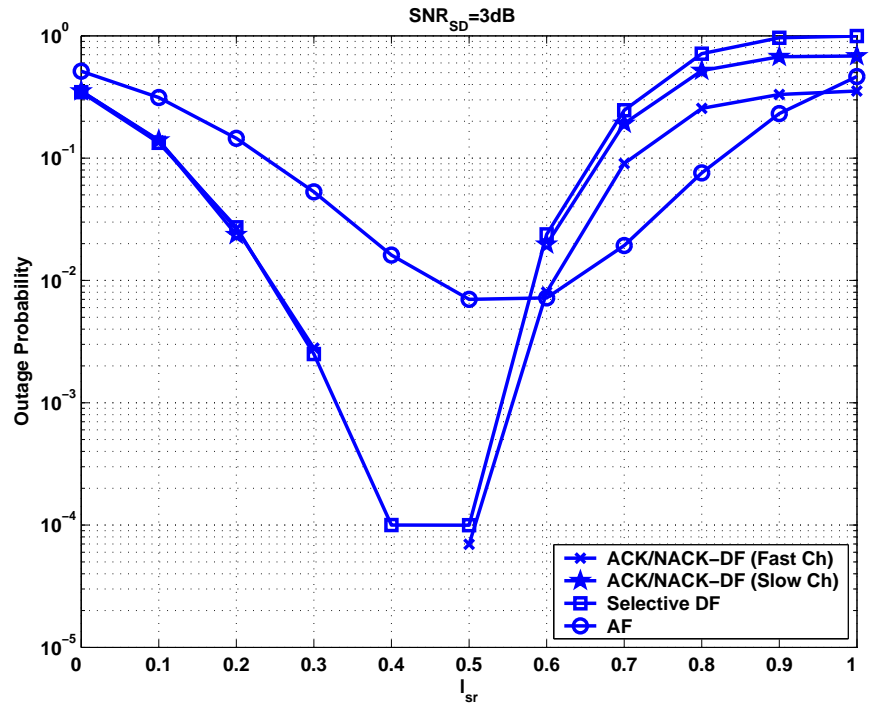

Figure 2. Outage probability versus $l_{S R}$ for $M_{\mathrm{S}}=M_{\mathrm{R}}=M_{\mathrm{D}}=2$, $K=2, L=3$, and the path loss exponent $\kappa=3$.

selective DF can be considered as the best DF relaying scheme as it does not involve the source during the relaying slots.

Now, we consider a multi-relay cooperative ARQ system where the source and the relays are equipped with two antennas, i.e., $M_{\mathrm{S}}=M_{\mathrm{R}}=2$, and the target rate is $\mathcal{R}=2$. In Fig. 3 and Fig. 4, we plot outage probability versus $\mathrm{SNR}_{\mathrm{SD}}$ at $l_{\mathrm{SR}}=0.5$ for one and two-relay cooperative ARQ systems ( $K=2$ and $K=3$ ). In both figures, we use conventional hybrid-ARQ as a reference to evaluate the behavior of the studied relaying schemes. In this conventional packet retransmission scheme, the packet is directly retransmitted by the source without relay assistance. In the following, we assume that the $\mathrm{S} \rightarrow \mathrm{D}$ link experiences short-term quasi-static fading. This corresponds to the best scenario for conventional hybridARQ where re-transmission rounds see different and independent channel realizations. In Fig. 3, all nodes are equipped with two antennas. In this case, for $K=2$, the SNR gain due to packet retransmission diversity in conventional hybrid-ARQ is less than $1 \mathrm{~dB}$ at $10^{-3}$ outage compared with $K=1$. However, when relays are incorporated in the network to play the role of packet re-transmitters, this gain is increased to more than $2 \mathrm{~dB}$ for $\mathrm{AF}$ relaying scheme and $5 \mathrm{~dB}$ for DF. This high diversity gain can be reached when the relay is at the optimal location $l_{\mathrm{SR}}=0.5$. Also, note that in conventional hybrid-ARQ transmission, the outage performance saturates after $K=2$ while for relaying transmission, the two-relay system has a diversity gain of $0.5 \mathrm{~dB}$ at $10^{-3}$ outage compared to the onerelay system. In Fig. 4, we notice that for overloaded multi-relay cooperative ARQ systems, i.e., $M_{\mathrm{S}}=M_{\mathrm{R}}=2$ and $M_{\mathrm{D}}=1$, the diversity gain is increased to $1 \mathrm{~dB}$ for $\mathrm{DF}$ relaying schemes. The throughput curves of balanced configuration with two relays are reported in Fig. 5. We observe that the benefits of incorporating relays in the network appear in the region of low to moderate SNR where multiple transmissions are required to help correct packets erroneously received during the first slot.

\section{CONCLusion}

In this paper, we investigated the issue of multi-relay communication over broadband MIMO channels. First of all, we derived an unified communication model for AF, Selective DF, and ACK/NACKaided DF. We presented a multi-slot communication model where 


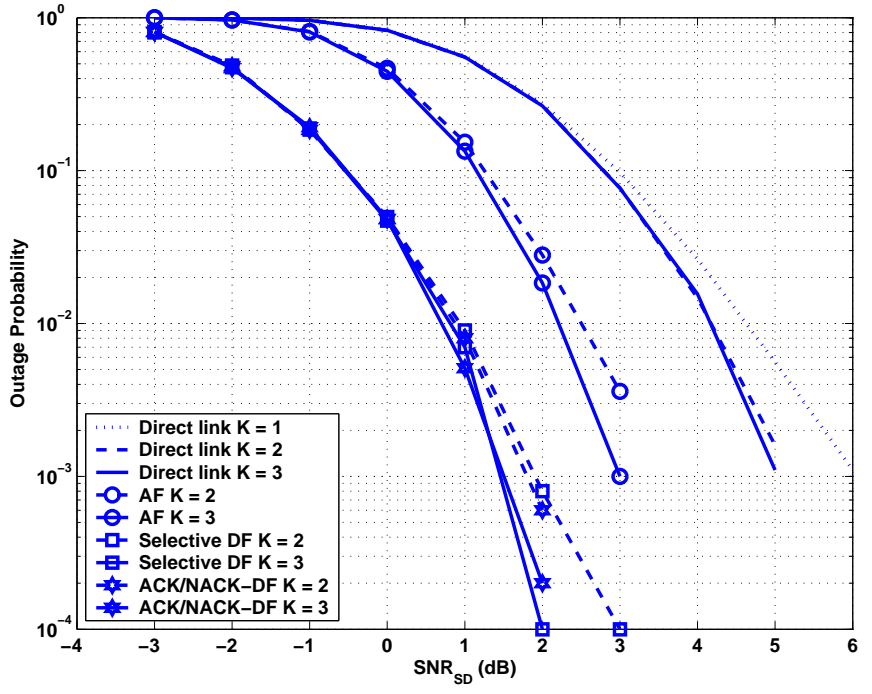

Figure 3. Outage probability versus $\mathrm{SNR}_{\mathrm{SD}}$ for $M_{\mathrm{S}}=M_{\mathrm{R}}=M_{\mathrm{D}}=2$, $l_{S R}=0.5, L=3$, and the path loss exponent $\kappa=3$.

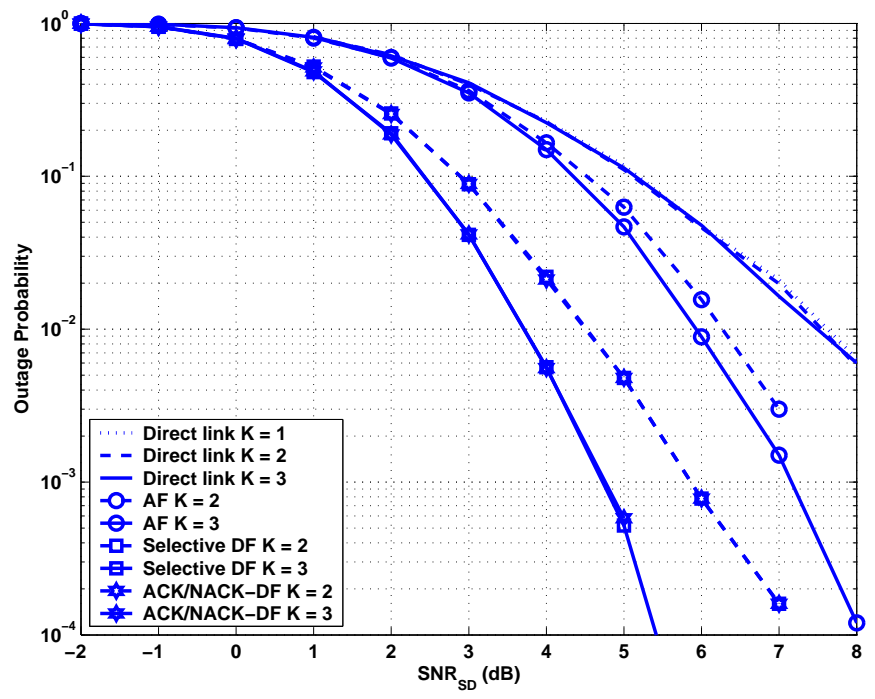

Figure 4. Outage probability versus $\mathrm{SNR}_{\mathrm{SD}}$ for $M_{\mathrm{S}}=M_{\mathrm{R}}=2, M_{\mathrm{D}}=1$, $l_{S R}=0.5, L=3$, and the path loss exponent $\kappa=3$.

transmission over relaying slots is translated into virtual receive antennas Then, we examined the outage probability of different studied relaying schemes. Using simulations, we showed that the studied relaying schemes outperform each other depending on relay locations. We also showed that the multi-relay-assisted communications provide better diversity gain than the conventional hybrid-ARQ.

\section{REFERENCES}

[1] S. Lin, D. J. Costello, and M. J. Miller, "Automatic repeat-request error control schemes," IEEE Communications Magazine, vol. 12, pp. 5-17, Dec. 1984

[2] G. Caire, and D. Tuninetti, "ARQ protocols for the Gaussian collision channel," IEEE Trans. Inf. Theory, vol. 47, no. 4, pp. 1971-1988, Jul. 2001.

[3] E. Zimmermann, P. Herhold, and G. Fettweis, "The impact of cooperation on diversity-exploiting protocols," IEEE VTC-spring, Milan, Italy, May 2004

[4] J. N. Laneman, G. W. Wornell, and D. N. C. Tse, "An efficient protocol for realizing cooperative diversity in wireless networks," IEEE International Symposium on Information Theory (ISIT), Washington, DC, June 2001.

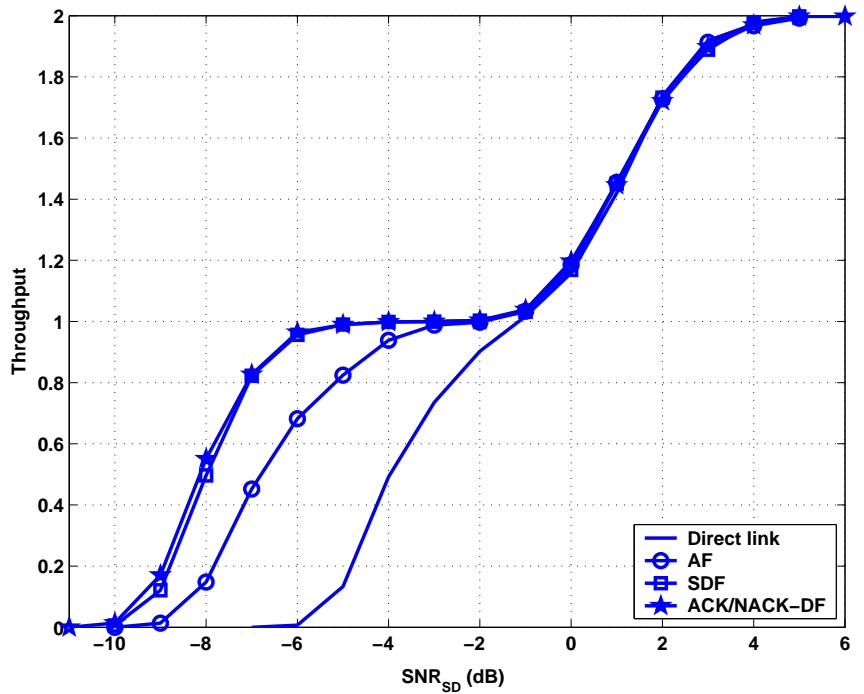

Figure 5. Throughput versus $\mathrm{SNR}_{\mathrm{SD}}$ for $M_{\mathrm{S}}=M_{\mathrm{R}}=M_{\mathrm{D}}=2, l_{S R}=$ $0.5, L=3$, and the path loss exponent $\kappa=3$.

[5] A. Sendonaris, E. Erkip, and B. Aazhang, "User cooperation diversity Part I \& Part II,” IEEE Trans. Commun., vol. 51, pp. 1927-1948, Nov. 2003.

[6] J. N. Laneman, D. Tse, and G. W. Wornell, "Cooperative diversity in wireless networks: Efficient protocols and outage behavior," IEEE Trans. Inform. Theory, vol. 50, no. 12, pp. 3062-3080, Dec. 2004.

[7] J. Boyer, D. D. Falconer, and H. Yanikomeroglu, "Multihop diversity in wireless relaying channels," IEEE Trans. on Comm., vol. 52, pp. 18201830, Oct. 2004.

[8] F. Atay Onat, H. Yanikomeroglu, and S. Periyalwar, "Relay-assisted spatial multiplexing in wireless fixed relay networks,", IEEE GLOBECOM, San Francisco, USA, Nov.- Dec. 2006.

[9] G. Yu, Z. Zhang, and P. Qiu, "Cooperative ARQ in wireless networks: Protocols description and performance analysis", IEEE International Conference on Communications (ICC), Istanbul, Turkey, June 2006.

[10] R. U. Nabar, F. W. Kneubiihler, and H. Boelcskei, "Performance limits of amplify-and-forward based fading relay channels", IEEE International Conference on Acoustics, Speech, and Signal Processing (ICASSP), Montreal, Canada, May 2004.

[11] Y. Zhang, H. H. Chen, and M. Guizani, Cooperative Wireless Communications, Auerbach Publications, 2009.

[12] B. Zhao and M. C. Valenti, "Practical relay networks: A generalization of hybrid-ARQ," IEEE J. Select. Areas. Comm., vol. 23, no. 1, Jan. 2005.

[13] H. V. Khuong and T. Le-Ngoc, "A Bandwidth-efficient Cooperative Relaying Scheme with Limited Feedback Information" 24th Biennial Symposium on Communication 2008, pp. 175-178, June 2008.

[14] H. Chafnaji, T. Ait-Idir, Halim Yanikomeroglu, and S. Saoudi, "Joint Turbo Equalization for Relaying Schemes over Frequency-Selective Fading Channels," in Proc, ACM Intern. Wireless Commun. Mobile Comput. Conf. (IWCMC'09), Leipzig, Germany, Jun. 2009.

[15] D. Tse, and P. Viswanath, "Fundamentals of wireless Communication," Cambridge University Press, May 2005.

[16] T. Ait-Idir and S. Saoudi, "Turbo Packet Combining Strategies for the MIMO-ISI ARQ Channel," IEEE Trans. Commun., vol. 57, no. 12, pp. 3782-3793, Dec. 2009.

[17] H. El Gamal, A. R. Hammons, Y. Liu, M. P. Fitz, and O. Y. Takeshita, "On the design of space-time and space-frequency codes for MIMO frequency-selective fading channels," IEEE Trans. Inform. Theory, vol. 49, no 9, pp. 2277-2292, Sep. 2003. 